\title{
New studies of allowed pion and muon decays
}

\author{
D. Počanić* ${ }^{*}$ A. Palladino, ${ }^{* \dagger}$ L. P. Alonzi*, V. A. Baranov**, W. Bertl ${ }^{\dagger}$, \\ M. Bychkov*, Yu.M. Bystritsky**, E. Frlež*, V.A. Kalinnikov**, \\ N.V. Khomutov**, A.S. Korenchenko**, S.M. Korenchenko**, M. Korolija ${ }^{*}$, \\ T. Kozlowski ${ }^{\S}$, N.P. Kravchuk**, N.A. Kuchinsky**, M.C. Lehman*, \\ D. Mekterović ${ }^{\ddagger}$, E. Munyangabe*, D. Mzhavia**,II, P. Robmann", \\ A.M. Rozhdestvensky**, S.N. Shkarovskiy**, U. Straumann", I. Supek ${ }^{\ddagger}$, \\ P. Truöl", Z. Tsamalaidzeף , A. van der Schaaf", E.P. Velicheva** and \\ V.P. Volnykh**
*Institute of Nuclear and Particle Physics, University of Virginia, Charlottesville, VA 22904, USA
${ }^{\dagger}$ Paul Scherrer Institut, Villigen PSI, CH-5232, Switzerland
${ }^{* *}$ Joint Institute for Nuclear Research, RU-141980 Dubna, Russia
$¥$ Institut Rudjer Bošković, HR-10000 Zagreb, Croatia
$\S_{\text {NCBJ National Centre for Nuclear Research, Otwock, Poland }}$
IInstitute for High Energy Physics, Tbilisi State University, GUS-380086 Tbilisi, Georgia
"Physik-Institut, Universität Zürich, CH-8057 Zürich, Switzerland

\begin{abstract}
Building on the rare pion and muon decay results of the PIBETA experiment, the PEN collaboration has undertaken a precise measurement of $B_{\pi \mathrm{e} 2} \equiv R_{\mathrm{e} / \mu}^{\pi}$, the $\pi^{+} \rightarrow \mathrm{e}^{+} v(\gamma)$ decay branching ratio, at the Paul Scherrer Institute, to reduce the present $40 \times$ experimental precision lag behind theory to $\sim 6-7 \times$. Because of large helicity suppression, $R_{\mathrm{e} / \mu}^{\pi}$ is uniquely sensitive to contributions from non- $(V-A)$ physics, making this decay a particularly suitable subject of study. Even at current precision, the experimental value of $B_{\pi \mathrm{e} 2}$ provides the most accurate test of lepton universality available. During runs in 2008-10, PEN has accumulated over $2 \times 10^{7} \pi_{\mathrm{e} 2}$ events; a comprehensive maximum-likelihood analysis is currently under way. The new data will also lead to improved precision of the earlier PIBETA results on radiative $\pi$ and $\mu$ decays.
\end{abstract}

Keywords: leptonic pion decays, muon decays, lepton universality

PACS: $13.20 . \mathrm{Cz}, 13.35 . \mathrm{Bv}$ 14.40.Be

Historically, the $\pi \rightarrow \mathrm{e} v$ (or $\pi_{\mathrm{e} 2}$ ) decay, provided an early confirmation of the $V-A$ nature of the electroweak interaction. Thanks to exceptionally well controlled theoretical uncertainties, its branching ratio is now understood at better than a part in $10^{4}$. The most recent independent theoretical calculations are in excellent agreement, and give:

$$
B_{\pi \mathrm{e} 2}^{\mathrm{SM}} \equiv R_{\mathrm{e} / \mu}^{\pi, \mathrm{SM}}=\left.\frac{\Gamma(\pi \rightarrow \mathrm{e} \bar{v}(\gamma))}{\Gamma(\pi \rightarrow \mu \bar{v}(\gamma))}\right|_{\text {calc }}= \begin{cases}1.2352(5) \times 10^{-4} & \text { Ref. [1], } \\ 1.2354(2) \times 10^{-4} & \text { Ref. [2], } \\ 1.2352(1) \times 10^{-4} & \text { Ref. [3], }\end{cases}
$$

where " $(\gamma)$ " indicates that radiative decays are included. Marciano and Sirlin [1] and Finkemeier [2] took into account radiative corrections, higher order electroweak leading logarithms, short-distance QCD corrections, and structure-dependent effects, while Cirigliano and Rosell [3] used two-loop chiral perturbation theory. A number of exotic 

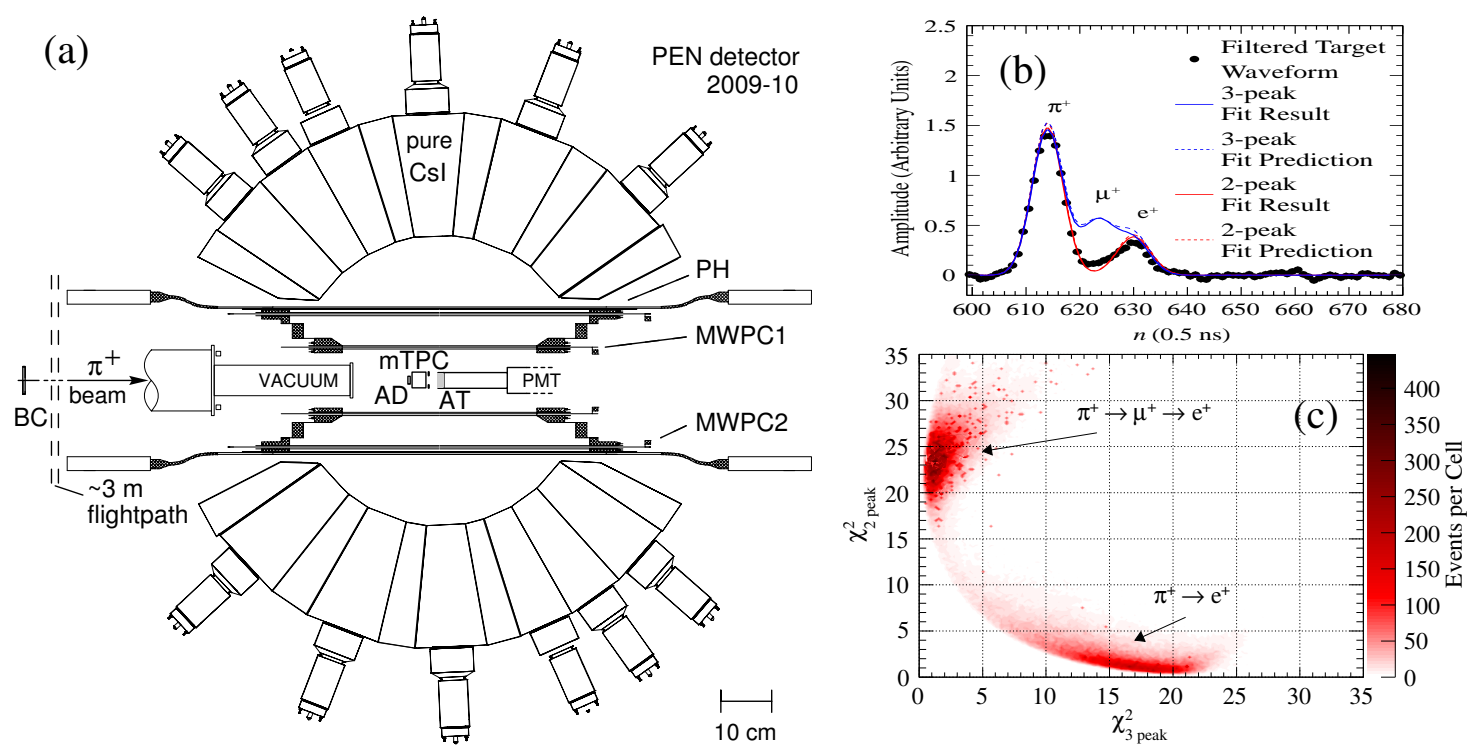

FIGURE 1. (a) Cross section drawing of the PEN detector system (details in text). (b) Filtered active target waveform for a $\pi \rightarrow \mathrm{e} v$ event showing a strongly preferred 2-peak fit. (c) Excellent $\chi^{2}$ separation of 2- and 3-peak filtered waveform fits (bottom).

processes outside of the current standard model (SM) can produce deviations from the above predictions based on lepton universality, mainly through induced pseudoscalar (PS) currents. Prime examples are: charged Higgs in theories with multiple Higgs bosons, PS leptoquarks in theories with dynamical symmetry breaking, classes of vector leptoquarks, parameters of certain SUSY partner particles, as well as non-zero neutrino masses and their mixing (Refs. [4, 5] give recent reviews of the subject). Thus, $\pi_{\mathrm{e} 2}$ decay complements direct searches for new physics at modern colliders.

The two most recent measurements of the branching ratio $[6,7]$ are mutually consistent and dominate the world average of $1.230(4) \times 10^{-4}$, which, however, trails the theoretical accuracy by a factor of 40 . The PEN experiment [8] is aiming to reach $(\Delta B / B)_{\pi \mathrm{e} 2} \simeq 5 \times 10^{-4}$, and in doing so, to set new limits on the above non-SM processes. PEN also aims to improve the PIBETA results for radiative decays $\pi^{+} \rightarrow \mathrm{e}^{+} v \gamma$ and $\mu^{+} \rightarrow \mathrm{e}^{+} v \bar{v} \gamma$. Meanwhile, PiENu [9], a complementary experiment currently under way at TRIUMF, has a similar goal for $(\Delta B / B)_{\pi \mathrm{e} 2}$.

The PEN experiment uses an upgraded version of the PIBETA detector system, described in detail in Ref. [10], and used in a series of rare pion and muon decay measurements $[11,12,13]$. The PEN apparatus, shown in Fig. 1(a), consists of a large-acceptance $(\sim 3 \pi \mathrm{sr})$ electromagnetic shower calorimeter (pure CsI, 12 radiation lengths thick) with non-magnetic tracking in concentric cylindrical multi-wire proportional chambers (MWPC1,2) and plastic scintillator hodoscope $(\mathrm{PH})$, surrounding a plastic scintillator active target (AT). Beam pions pass through an upstream detector (BC), lose energy in the active degrader (AD), are tracked in a mini time projection chamber (mTPC), and stop in the AT. Signals from the beam detectors are digitized in waveform digitizers, running at $2 \mathrm{GS} / \mathrm{s}$ for $\mathrm{BC}, \mathrm{AD}$, and $\mathrm{AT}$, and at $250 \mathrm{MS} / \mathrm{s}$ for $\mathrm{mTPC}$.

The $\pi_{\mathrm{e} 2}$ branching ratio will be evaluated by normalizing the observed yield of $\pi \rightarrow \mathrm{e} v$ 
decays to the number of sequential decays $\pi \rightarrow \mu \rightarrow \mathrm{e}$, within a 250 ns gate starting some $40 \mathrm{~ns}$ before the pion stop time [8]. Assignment of detected events to either of the two processes, or to a background process, is made within a comprehensive, blind and unbinned maximum likelihood analysis (MLA) [14]. Key to achieving the goal uncertainty is in the control of the systematics.

One among many powerful tools available to develop reliable probability density functions for the MLA is provided by the beam counter waveform (wf) digitizer data, helping us to obtain clean samples of $\pi_{\mathrm{e} 2}$ events (two pulses in the AT-wf) and sequential $\pi \rightarrow \mu \rightarrow$ e decay events (three pulses in the AT-wf). The procedure and results are summarily illustrated in Fig. 1(b,c). Another key component in the analysis is the comprehensive GEANT4 Monte Carlo simulation of the experiment that produces synthetic data fully equivalent to the measured data [15].

Furthermore, thanks to lower and better controlled backgrounds, the PEN data will enable us to improve on the precision of the previous PIBETA results on pion [13] and muon $[16,17]$ radiative decays, both of which are sensitive to non- $(V-A)$ interactions. Besides providing the most stringent limit on tensor interactions to date [18], the PIBETA $\pi \rightarrow \mathrm{e} v \gamma$ results [13] also furnish fundamental inputs for chiral perturbation theory related to pion structure. On the other hand, muon radiative decay, being largely free from hadronic corrections, provides model-independent information on non- $(V-A)$ admixtures in the lagrangian.

Three PEN data runs have been completed, in 2008, 2009, and 2010, respectively, collecting over $20 \mathrm{M} \pi_{\mathrm{e} 2}$ events. Comprehensive data analysis, focusing on control of systematics, is currently under way.

This work has been supported by grants from the US National Science Foundation (most recently PHY-0970013), the Paul Scherrer Institute, and the Russian Foundation for Basic Research (Grant 08-02-00652a).

\section{REFERENCES}

1. W.J. Marciano and A. Sirlin, Phys. Rev. Lett. 71 (1993) 3629.

2. $\quad$ M. Finkemeier, Phys. Lett. B 387 (1996) 391.

3. V. Cirigliano and I. Rosell, Phys. Rev. Lett. 99 (2007) 231801.

4. $\quad$ M. Raidal, A. van der Schaaf, I. Bigi, et al., Eur. Phys. J C 57 (2008) 13.

5. D. Bryman, W.J. Marciano, R. Tschirhart, T. Yamanaka, Ann. Rev. Nucl. Part. Sci. 61 (2011) 331.

6. D.I. Britton, S. Ahmad, D.A. Bryman, et al., Phys. Rev. Lett. 68 (1992) 3000.

7. G. Czapek, A. Federspiel, A. Flükiger, et. al., Phys. Rev. Lett. 70 (1993) 17.

8. "A precise measurement of the $\pi^{+} \rightarrow e^{+} v$ branching ratio," PSI experiment proposal R-05-01, http://pen.phys.virginia.edu/proposal/pen_proposal.pdf (2006).

9. PiENu: http://pienu.triumf.ca, and A. Sher, elsewhere in these Proceedings.

10. E. Frlež, D. Počanić, K. Assamagan, et al., Nucl. Inst. and Meth. A 526 (2004) 300.

11. D. Počanić, E. Frlež, V.A. Baranov, et al., Phys. Rev. Lett. 93 (2004) 181803.

12. E. Frlež, D. Počanić, V.A. Baranov, et al., Phys. Rev. Lett. 93 (2004) 181804.

13. M. Bychkov, D. Počanić, B.A. VanDevender, et al., Phys. Rev. Lett. 103 (2009) 051802.

14. A. Palladino, $\mathrm{PhD}$ thesis, University of Virginia (2012).

15. L.P. Alonzi, PhD thesis, University of Virginia (2012).

16. B.A. VanDevender, PhD thesis, University of Virginia (2006).

17. E. Munyangabe, $\mathrm{PhD}$ thesis, University of Virginia (2012), and article in preparation.

18. T. Bhattacharya, V. Cirigliano, S.D. Cohen, et al., Phys. Rev. D 85 (2012) 054512. 